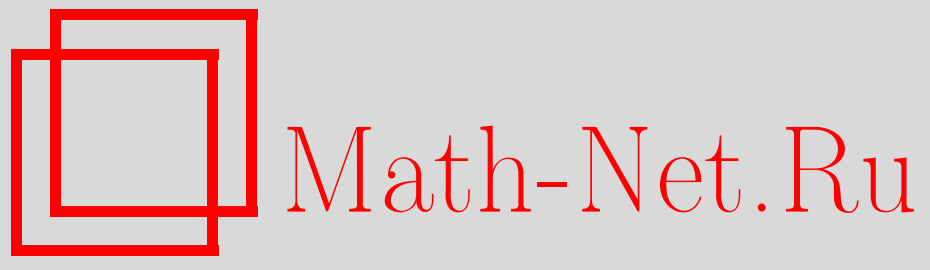

Ф. Шаров, Прямоугольник из квадратов, Квант, 2019, номер 3, 10-14

DOI: https://doi.org/10.4213/kvant20190302

Использование Общероссийского математического портала Math-Net.Ru подразумевает, что вы прочитали и согласны с пользовательским соглашением http://www.mathnet.ru/rus/agreement

Параметры загрузки:

IP : 3.89 .197 .203

26 апреля 2023 г., 14:57:16

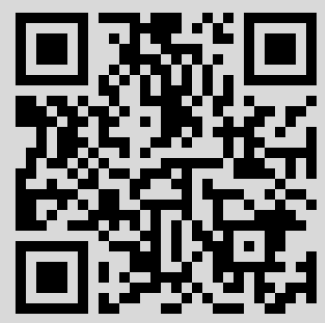




\section{Прямоугольник из квадратов}

Ф.ШАРOB

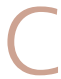
ДАВНИХ ВРЕМЕНЛЮДИ ЗАНИМАЛИСЬ задачами на разрезания. Можно ли разрезать определенную фигуру на какието другие (например, на квадраты)? Можно ли из некоторого набора фигур составить другую заданную фигуру (например, правильный треугольник)? В этой статье мы докажем один из классических результатов в этой области математики - теорему Дена.

Теорема 1 (М.Ден, 1903). Если прямоугольник разрезан на квадраты (не обязательно равные), то отношение длин его сторон ращионально.

Читатель, конечно, без труда сможет догадаться, как разрезать прямоугольник с рациональным отношением длин сторон на квадраты (рис.1). Содержательным

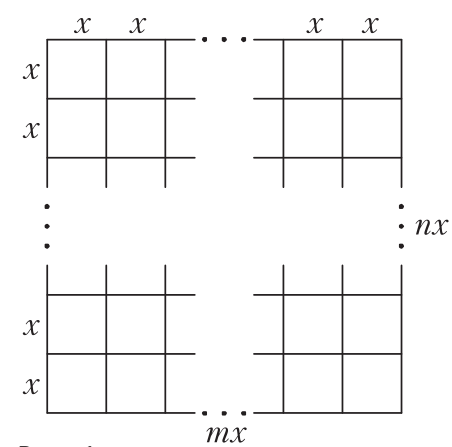

РИс. 1

является лишь вопрос невозможности разрезаний в остальных случаях.

Например, у прямоугольника вида $(a+b \sqrt{2}) \times(c+d \sqrt{2})$, где $a, b, c, d$ рациональны, длины сторон и их отношения уже могут быть иррациональны. На этом простейшем частном случае уже можно продемонстрировать все основные идеи, лежащие в основе доказательства теоремы 1. В дальнейшем для удобства латинские бук-

DOI: https://doi.org/10.4213/kvant20190302 вы $a, b, c, d$ и эти же буквы с индексами будут означать ращиональные числа, а все числа вида $a+b \sqrt{2}$ будем называть хороиими.

\section{Теорема Дена для хороших чисел}

Сначала докажем теорему для частного случая, когда стороны разрезаемого прямоугольника и стороны всех квадратов являются хорошими числами.

Прежде всего рассмотрим пример: прямоугольник $1 \times \sqrt{2}$. Решим следующую задачу.

Задача 1. Можно ли прямоугольник $1 \times \sqrt{2}$ разрезать на квадраты с рациональными сторонами? А со сторонами, которые либо рациональны, либо имеют вид $b \sqrt{2}$ ? А со сторонами, которые являются произвольными хорошими числами?

Решение. ${ }^{1}$ Oтвет на первые два вопроса: нет. Решение для третьего вопроса приведено в конце журнала.

Ответим на первый вопрос. Предположим, что прямоугольник $1 \times \sqrt{2}$ разрезан на квадраты с рациональными сторонами. Рассмотрим сторону, равную $\sqrt{2}$. Сумма примыкающих к ней сторон квадратов должна равняться $\sqrt{2}$. Сумма рациональных чисел не может быть равна иррациональному, следовательно, такое разрезание невозможно.

Ответим на второй вопрос. Предположим, что прямоугольник $1 \times \sqrt{2}$ разрезан на квадраты со сторонами вида $a$ или $b \sqrt{2}$. Его площадь равна $\sqrt{2}$. Площади квадратов имеют вид либо $a^{2}$, либо $2 b^{2}$.

${ }^{1}$ Решение задачи 1 , а также приведенное ниже доказательство невозможности разрезания прямоугольника $1 \times(1+\sqrt{2})$ на квадраты и решение задачи 3 (приведенное в конце журнала) написаны Е.Алиевой. 
Площадь прямоугольника равна сумме площадей квадратов. Получаем противоречие, так как иррациональное число не может быть равно сумме рациональных. Здесь мы опирались на аддитивность площади: площадь целого равна сумме площадей частей.

Покажем теперь, что прямоугольник $1 \times(1+\sqrt{2})$ нельзя разрезать на квадраты c хорошими сторонами. Для этого нам понадобится определение сопряженного числа.

Определение 1. Число $\bar{s}=a-b \sqrt{2}$ называется сопряженным к числу $s=a+$ $+b \sqrt{2}$.

Задача 2. Докажите, что сопряженное к сумме хороших чисел равно сумме сопряженных, а сопряженное к произведению произведению сопряженных.

Итак, предположим, что прямоугольник $1 \times(1+\sqrt{2})$ разрезан на $n$ квадратов со сторонами $a_{1}+b_{1} \sqrt{2}, a_{2}+b_{2} \sqrt{2}, \ldots, a_{n}+$ $+b_{n} \sqrt{2}$. Площадь данного прямоугольника равна сумме площадей рассматриваемых $n$ квадратов, т.е.

$$
\begin{aligned}
1+\sqrt{2}= & \left(a_{1}+b_{1} \sqrt{2}\right)^{2}+ \\
& +\left(a_{2}+b_{2} \sqrt{2}\right)^{2}+\ldots+\left(a_{n}+b_{n} \sqrt{2}\right)^{2} .
\end{aligned}
$$

Найдем сопряженные к обеим частям полученного равенства. Сопряженным к числу $1+\sqrt{2}$ является число $1-\sqrt{2}$. По утверждению задачи 2 получаем, что сопряженным к числу

$\left(a_{1}+b_{1} \sqrt{2}\right)^{2}+\left(a_{2}+b_{2} \sqrt{2}\right)^{2}+\ldots+\left(a_{n}+b_{n} \sqrt{2}\right)^{2}$

является число

$\left(a_{1}-b_{1} \sqrt{2}\right)^{2}+\left(a_{2}-b_{2} \sqrt{2}\right)^{2}+\ldots+\left(a_{n}-b_{n} \sqrt{2}\right)^{2}$.

Получаем, что

$$
\begin{aligned}
1-\sqrt{2}= & \left(a_{1}-b_{1} \sqrt{2}\right)^{2}+ \\
& +\left(a_{2}-b_{2} \sqrt{2}\right)^{2}+\ldots+\left(a_{n}-b_{n} \sqrt{2}\right)^{2} .
\end{aligned}
$$

Число $1-\sqrt{2}$ - отрицательное, а правая часть тождества - сумма неотрицательных. Получаем противоречие. Значит, прямоугольник $1 \times(1+\sqrt{2})$ нельзя раз- резать на квадраты с хорошими сторонами.

Заметим, что, например, сопряженное к числу $2+\sqrt{2}$ положительно. Поэтому только что использованный метод доказательства невозможности разрезания прямоугольника $1 \times(1+\sqrt{2})$ неприменим для доказательства невозможности разрезания прямоугольника $1 \times(2+\sqrt{2})$, а его, согласно теореме Дена, тоже нельзя разрезать на квадраты с хорошими сторонами. Обойти это препятствие можно при помощи следующего обобщения понятия площади.

Определение 2. Пусть $x$ - некоторое действительное число. Назовем $x$-nлощадъю прямоугольника $(a+b \sqrt{2}) \times(c+d \sqrt{2})$ число $(a+b x)(c+d x)$.

Например, $\sqrt{2}$-площадь - это обычная площадь такого прямоугольника, a $(-\sqrt{2})$ площадь - сопряженное к ней число.

Задача 3. Найдите все прямоугольники вида $(a+b \sqrt{2}) \times(c+d \sqrt{2}), x$-площади которых неотрицательны при всех $x$.

Лемма 1 (аддитивность $x$-площади). Если прямоугольник разрезан на конечное число прямоугольников, стороны которых - хорошие числа, то для любого действительного числа $x$ х-площадь разрезаемого прямоугольника равна сумме х-площадей прямоугольников, на которые он разрезан.

Доказательство. Нетрудно убедиться, что сумма $x$-площадей двух прямоугольников со сторонами вида $a+b \sqrt{2}$, имеющих общую сторону, равна $x$-площади их объединения.

Действительно, пусть имеется прямоугольник с $x$-площадью $S$, который состоит из двух прямоугольников с $x$-площадями

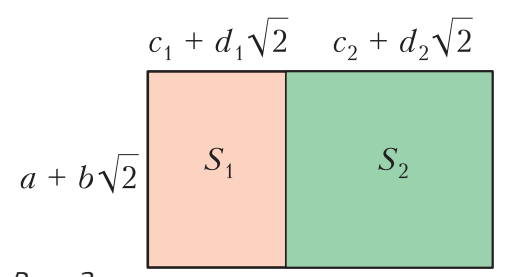

Pnc. 2 
$S_{1}$ и $S_{2}$ (рис.2). Тогда

$S_{1}+S_{2}=$

$$
\begin{array}{r}
=(a+b x)\left(c_{1}+d_{1} x\right)+(a+b x)\left(c_{2}+d_{2} x\right)= \\
=(a+b x)\left(\left(c_{1}+c_{2}\right)+\left(d_{1}+d_{2}\right) x\right)=S .
\end{array}
$$

Пусть теперь количество прямоугольников в разрезании больше двух. Продолжим каждый разрез, как показано на рисунке 3. Тогда каждый прямоугольник

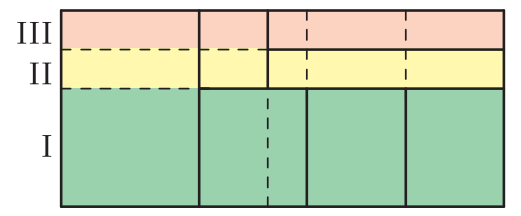

Pис. 3

нового разрезания будет также иметь стороны вида $a+b \sqrt{2}$. Рассмотрим горизонтальные слои из последовательно приложенных друг к другу по общей стороне прямоугольников (I, II, III на рисунке 3). Используя уже доказанное свойство аддитивности $x$-площади двух прямоугольников с общей стороной, методом математической индукции легко доказывается, что $x$-площадь любого такого слоя равна сумме $x$-площадей прямоугольников, составляющих этот слой. Теперь уже эти слои приложим друг к другу и применим только что доказанное утверждение об аддитивности $x$-площади ряда прямоугольников. Получим, что $x$-площадь разрезаемого прямоугольника равна сумме $x$-площадей горизонтальных слоев. Эта сумма равна сумме $x$-площадей всех прямоугольников нового разрезания. С другой стороны, каждый прямоугольник старого разрезания (так же, как и большой прямоугольник) составлен из прямоугольников нового разрезания, в один или несколько слоев. Таким образом, сумма $x$-площадей прямоугольников старого разрезания равна $x$ площади исходного прямоугольника.

Задача 4. Докажите, что отношение двух хороших чисел - хорошее число.

Теорема 2 (частный случай теоремы Дена). Прямоугольник с хорошими длинами сторон можно разрезать на квадрать с хорошими длинами сторон тогда и толь- ко тогда, когда отношение его сторон рачионально.

Доказательство. Предположим, что отношение сторон иррационально, а разрезание существует. Поделим стороны прямоугольника и всех квадратов на меньшую сторону прямоугольника. По задаче 4 все стороны останутся хорошими. В частности, полученный прямоугольник будет иметь вид $1 \times(c+d \sqrt{2})$. Его $x$-площадь равна $c+d x$. Так как отношение сторон иррационально, то $d \neq 0$. Значит, $x$-площадь отрицательна при некотором $x$. В то же время $x$-площадь любого квадрата со стороной $a+b \sqrt{2}$ равна $(a+b x)^{2}$, что неотрицательно для любого $x$. Получили противоречие с леммой 1 : сумма неотрицательных чисел не может равняться отрицательному.

Задача 5. Пусть $z$ - произвольное иррациональное число. Можно ли прямоугольник $1 \times z$ разрезать на квадраты со сторонами вида $a+b z$ ?

Подсказка: попробуйте модифицировать понятие $x$-площади так, чтобы оно стало определено для прямоугольника и квадратов, рассматриваемых в задаче.

\section{Доказательство теоремы Дена в общем случае}

Для доказательства теоремы Дена в общем случае определение $x$-площади нам уже не годится: ведь она определена только для хороших чисел, а теперь у нас в разрезании могут присутствовать квадраты с какими угодно сторонами (например, $\sqrt{3}, \pi, \sqrt[3]{2}$ и так далее).

Далее в этом разделе мы считаем, что прямоугольник $s_{0} \times t_{0}$ разрезан на прямоугольники $s_{1} \times t_{1}, s_{2} \times t_{2}, \ldots, s_{N} \times t_{N}$, причем $s_{0}$ и $t_{0}$ несоизмеримы, т.е. их отношение иррационально.

Лемма 2. Обозначим

$$
P=\left\{s_{0}, t_{0}, s_{1}, t_{1}, \ldots, s_{N}, t_{N}\right\} .
$$

Тогда можно выбрать такие числа $e_{1}, e_{2}, \ldots, e_{n} \in P$, чтобы любое число $p \in P$ единственным образом представлялось в виде

$$
p=a s_{0}+b t_{0}+a_{1} e_{1}+a_{2} e_{2}+\ldots+a_{n} e_{n} .
$$


Например, для разрезания на рисунке 4, где $s_{0}=1$ и $t_{0}=2+\sqrt{2}$, необходимо выбрать лишь одно число (либо $e_{1}=\sqrt{3}$, либо $\left.e_{1}=2+\sqrt{2}-\sqrt{3}\right)$.

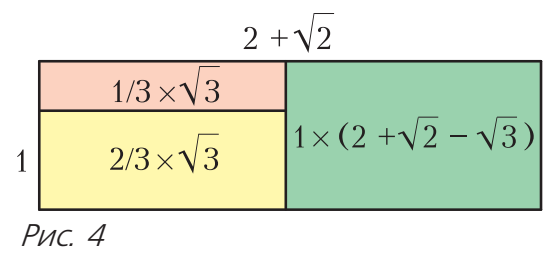

Доказательство. Выпишем в строку длины сторон прямоугольников разрезания, начиная с $s_{0}$ и $t_{0}$. Так, для разрезания на рисунке 4 получится такая строка:

$$
\begin{gathered}
s_{0}=1, t_{0}=2+\sqrt{2}, s_{1}=1 / 3, t_{1}=\sqrt{3}, \\
s_{2}=2 / 3, t_{2}=\sqrt{3}, s_{3}=1, t_{3}=2+\sqrt{2}-\sqrt{3} .
\end{gathered}
$$

Теперь ${ }^{2}$ подчеркнем в этом списке те числа, которые не представляются в виде суммы предыдущих с рациональными коэффициентами (сейчас поясним, что это значит). Например, $s_{0}$ мы подчеркнем (предыдущих чисел вовсе нет). Число $t_{0}$ мы тоже подчеркнем, так как оно несоизмеримо с $s_{0}$ и, следовательно, не представимо в виде $a_{0} s_{0}$. Далее, если $s_{1}$ представляется в виде $a_{0} s_{0}+b_{0} t_{0}$, то $s_{1}$ не подчеркиваем, а если не представляется, то подчеркиваем. Аналогично, если $t_{1}$ представляется в виде $a_{0} s_{0}+b_{0} t_{0}+a_{1} s_{1}$, то $t_{1}$ не подчеркиваем, а если не представляется, то подчеркиваем. И так далее. В нашем случае получится такое:

$1, \underline{2}+\sqrt{2}, 1 / 3, \underline{\sqrt{3}}, 2 / 3, \sqrt{3}, 1,2+\sqrt{2}-\sqrt{3}$.

Докажем теперь, что всякое число из этого списка единственным образом представляется как сумма подчеркнутых чисел с рациональными коэффициентами. Ясно, что неподчеркнутое число представляется нужным образом через подчеркнутые. Подчеркнутое тоже представляется: оно равно само себе. Если же такое представление не единственно, то, вычитая одно

\footnotetext{
2 Дальнейший текст доказательства леммы 2 с незначительными изменениями заимствован из статьи Д.Фукса «Можно ли из тетраэдра сделать куб?» [4].
}

представление из другого, мы видим, что сумма неких подчеркнутых чисел с рациональными коэффициентами равна 0. Но это позволяет выразить, опять-таки в виде суммы с рациональными коэффициентами, одно из подчеркнутых чисел через предыдущие подчеркнутые числа, а значит, мы зря его подчеркнули.

Bce подчеркнутые числа, начиная с третьего, и будут искомым набором $e_{1}, e_{2}, \ldots, e_{n}$.

Зафиксируем набор чисел $s_{0}, t_{0}, e_{1}, e_{2}, \ldots, e_{n}$ из леммы 2. Он называется базисом.

Определение 3. Пусть $y$ - некоторое действительное число. Назовем $y$-nлощ $a^{-}$ дъю прямоугольника со сторонами

$$
\begin{aligned}
& a s_{0}+b t_{0}+a_{1} e_{1}+a_{2} e_{2}+\ldots+a_{n} e_{n} \\
& \text { и } c s_{0}+d t_{0}+c_{1} e_{1}+c_{2} e_{2}+\ldots+c_{n} e_{n}
\end{aligned}
$$

число $(a+b y)(c+d y)$.

Например, $y$-площадь разрезаемого прямоугольника $s_{0} \times t_{0}$ равна $y$.

Обратите внимание, что это определение не эквивалентно определению $x$-площади выше! Скажем, для прямоугольника на рисунке $4 x$-площадь равна $2+x$, а $y$-площадь равна $y$. Если подставить в качестве $x$ и $y$ одинаковые числа, то могут получиться разные результаты.

Лемма 3. Любой квадрат в разрезании прямоугольника $s_{0} \times t_{0}$ имеет неотрицательную у-площадь.

Доказательство. Сторона любого квадрата в разрезании прямоугольника $s_{0} \times t_{0}$ записывается в виде $a s_{0}+b t_{0}+a_{1} e_{1}+$ $+a_{2} e_{2}+\ldots+a_{n} e_{n}$. Тогда его $y$-площадь равна $(a+b y)^{2}$, что неотрицательно при любом действительном $y$.

Лемма 4. Для любого у у-площадь разрезаемого прямоугольника $s_{0} \times t_{0}$ равна сумме у-плошадей прямоугольников, на которые он разрезан.

Доказательство леммы 4 дословно повторяет доказательство леммы 1 с той лишь разницей, что нужно заменить в нем $x$ площадь на $y$-площадь, а также заменить все числа вида $a+b \sqrt{2}$ на соответствующие числа вида $a s_{0}+b t_{0}+a_{1} e_{1}+a_{2} e_{2}+\ldots$ $\ldots+a_{n} e_{n}$ (в том числе и на рисунке 2). 
Доказательство теоремы 1. Пусть прямоугольник $s_{0} \times t_{0}$ разрезан на квадраты, причем $s_{0}$ и $t_{0}$ несоизмеримы. По определению его $y$-площадь равна $y$. Это число отрицательно при $y<0$. В то же время $y$-площадь любого квадрата неотрицательна по лемме 3. Получили противоречие с леммой 4: сумма неотрицательных чисел не может равняться отрицательному.

В заключение отметим, что $y$-площадь это не просто олимпиадный трюк. В математике есть важное понятие меры. Мерами являются, например длина отрезков на прямой; площадь (обыкновенная, а не $y$-площадь) фигур на плоскости; объем тел в пространстве. Из двух основных свойств меры - аддитивности и неотрицательности - наша $y$-площадь обладает только первым.

Если свойство неотрицательности не требуется, вместо слова «мера» используется термин «заряд». Вот примером заряда и является $y$-площадь.

\section{Литература}

1. М.Скопенков, О.Малиновская, С.Дори ченко, Ф.Шаров. Собери квадрат. В сборнике: Элементы математики в задачах: через олимпиады и кружки - к профессии. - М.: МЦНМО, 2018.

2. М.Скопенков, О.Малиновская, С.Дориченко. Собери квадрат. - «Квант», 2015, №2 .

3. М.Скопенков, М.Прасолов, С.Дориченко. Разрезания металлического прямоугольника. - «Квант», 2011, №3.

4. Д.Фукс. Можно ли из тетраэдра сделать куб? - «Квант», 1990, №11.

5. Ф.Шаров. Разрезания прямоугольника на прямоугольники с заданными отношениями сторон. - Математическое просвещение, сер. 3, вып. 20 (2016), с.200-216.

6. И.М.Яглом. Как разрезать квадрат? - М.: Наука, 1968.

\section{Вниманию наших читателей!}

В предыдущем номере журнала в статье «Объять необъятное ...» было допущено несколько опечаток. Необходимо сделать следующие исправления.

\begin{tabular}{|c|c|c|}
\hline Где: & Было: & Должно быть: \\
\hline $\begin{array}{l}\text { с.4, левая колонка, } \\
6 \text {-я строка снизу }\end{array}$ & немного & намного \\
\hline $\begin{array}{l}\text { с.7, левая колонка, } \\
11 \text {-я строка снизу }\end{array}$ & таких & малых \\
\hline $\begin{array}{l}\text { c.9, правая колонка, } \\
\text { последний абзац }\end{array}$ & $E_{1}=85$ МэВ/нуклон & $E_{1}=8,5 \mathrm{MэВ/нуклон}$ \\
\hline $\begin{array}{l}\text { с.10, левая колонка, } \\
\text { 2-й абзац }\end{array}$ & $\rho_{\mathrm{cp}} \sim 5 \cdot 10^{30} \mathrm{r} / \mathrm{cm}^{3}$ & $\rho_{\mathrm{cp}} \sim 5 \cdot 10^{-30} \mathrm{\Gamma} / \mathrm{cm}^{3}$ \\
\hline $\begin{array}{l}\text { c. } 10, \text { левая колонка, } \\
\text { предпоследний абзац }\end{array}$ & $\frac{c}{H} \sim 10^{6} \mathrm{M}$ & $\frac{c}{H} \sim 10^{26} \mathrm{M}$ \\
\hline $\begin{array}{l}\text { с. } 11 \text {,правая колонка, } \\
\text { первый абзац }\end{array}$ & $x=2$ & $x=1$ \\
\hline $\begin{array}{l}\text { с.12, правая колонка, } \\
\text { первый абзац }\end{array}$ & $\begin{array}{l}T_{\mathrm{C}}=T_{3} \cdot 4 \cdot 4 \cdot\left(\frac{l_{\mathrm{C} 3}}{2 R_{\mathrm{C}}}\right)= \\
=20 T_{3}=6000 \mathrm{~K}\end{array}$ & $\begin{array}{l}T_{\mathrm{C}}=T_{3} \cdot(4 \cdot 4)^{1 / 4} \cdot\left(\frac{l_{\mathrm{C} 3}}{2 R_{\mathrm{C}}}\right)^{1 / 2}= \\
=20 T_{3}=6000 \mathrm{~K}\end{array}$ \\
\hline
\end{tabular}

